doi:10.13108/2021-13-1-77

\title{
RELATIONS BETWEEN LENGTH AND INSTABILITY OF TUBULAR EXTREMAL SURFACES
}

\author{
N.M. POLUBOYAROVA
}

\begin{abstract}
In the paper we study surfaces being extremals of the potential energy functional. In our case, the potential energy is the sum of two functionals, one being a functional of the area type, and the other being a functional of the volume density of forces. Extremal surfaces are stable if the second variation of the functional is sign-definite, otherwise they are unstable. In order to obtain the instability, we impose additional conditions on the surface and integrands, then we apply the properties of positive definite symmetric matrices, employ the Kronrod-Federer formula, the Cauchy-Bunyakovsky inequality, and the Weingarten homomorphism estimate. This allows us to estimate the second variation of the functional. Such technique, being a developing of an approach proposed by V.A. Klyachin, allows us to obtain conditions ensuring the instability. We establish that the length of the tubular extremal surface can be estimated in terms of the minimal and maximal $(n-1)$-dimensional measure of the cross-sections of the surface by hyperplanes. The obtained statement means that too long tubes with a non-zero mean curvature are unstable. The physical aspects of this phenomenon were considered in a work by V.A. Saranin.
\end{abstract}

Keywords: variation of a functional, extremal surface, area-type functional, volume density functional, potential energy functional, stability, instability, tubular surface, hyperplane, measure of surface section, length of tubular surface.

Mathematics Subjects Classifications: 53A10, 30C70, 31A15

\section{INTRODUCTION}

The studies presented in this paper can be regarded as some generalizations of issues on stability and instability of minimal surfaces. The problem of stability of submanifolds of zero mean curvature in Riemannian manifolds was studied rather deeply. For instance, works by J.L. Barbosa and M.P. do Carmo, B. Lawson, A.V. Pogorelov, J. Simons, A.A. Tuzhilin, A.T. Fomenko and others were devoted to this problem. The study of a similar problem for pseudo-Riemannian manifolds was initiated rather recently, but there are already interesting approaches and results. J.L. Barbosa and M.P. do Carmo showed that an oriented minimal surface in $\mathbb{R}^{3}$, for which the area of the image under the Gauss mapping is less than $2 \pi$, is stable. M.P. do Carmo and C.K. Peng provided a generalization of the Bernstein problem in terms of stability: a plane is the only complete stable minimal surface in $\mathbb{R}^{3}$, while in the Lobachevskii space there exist one-parametric families of such surfaces. In work by A.A. Tuzhilin such families are described in details by means of the notion of "index", while the index of minimal revolution surfaces in Lobachevskii space is studied by the method of Fourier series. Since the definitions of stability of minimal surfaces given by various authors differ, we specify the terminology we use.

N.M. Poluboyarova, Relations Between length and instability of tubular extremal surFACES.

(C) Poluboyarova N.M. 2021.

The work is supported by the Mathematical Center in Academgorodok, agreement with the Ministry of Science and Higher Education of Russian Federation no. 075-15-2019-1613.

Submitted August 30, 2020. 
The minimal surfaces are the extremals of the area functional and their stability is defined as a positive definiteness of the second variation of this area functional. If one considers surfaces having some density and being under external forces, the functional changes. As examples of such surfaces physical equilibrium liquids in a gravitational field with a potential [1] or awning covers. From the physical point of view, the stability of the mechanical system means a positive definiteness of the second variation of the potential energy of the system.

In our case the potential energy is described by the sum of two functionals, one being an area-type functional, while the other is the functional of force volume density. To obtain the formulae for the first and second variations of the potential energy function we employ the method proposed in [2]. Its matter is that the surface is deformed along unit normals by means of a perturbing function with a compact support on the surface. We apply this method for the hypersurfaces obtained by embedding the manifold in $\mathbb{R}^{n+1}$. Earlier, this method was used for the surfaces of zero mean curvature in curved Lorentz products in paper [3] by V.A. Klyachin and V.M. Miklyukov as well as for the surfaces of a prescribed mean curvature in work [4] by V.A. Klyachin. As a visualization of this method, we can suggest the picture of magnetic liquid in a field with a potential provided in [5].

Once we obtain the second variation of the potential energy functional, we note that subject to the sign-definiteness of the matrix of the second derivatives of the integrand in the area type functional, one needs to consider the problem on either minimum or maximum of the functional. Examples were given in [6]. In this work we consider positive definite matrices.

Studying the stability and instability of the extremals of the potential energy, we employ various methods: capacity technique, mapping with a bounded distortion, surfaces of $G$-parabolic type. In the present work we apply the method of sectioning the surfaces by special planes and for estimating the length of the tube, we employ the quotient of the maximal and minimal measures of the sections of the surface. For instance, in work [5] by V.A. Saranin it was experimentally proved that a liquid cylinder is unstable if its height is greater than the length of the base. V.A. Klyachin in [4] stated that too lengthy tube of constant mean curvature are unstable. On the base of these works, there was proposed a conjecture that a too lengthy extremal tubular surface is also unstable. This conjecture is proved in the present work.

The results of the present work were announced on the International Conference "Complex Analysis and Geometry" in Ufa [8].

\section{FUNCTIONAL AND ITS VARIATIONS}

Let $M$ be an $n$-dimensional connected oriented manifold of class $C^{2}$. We consider an oriented hypersurface $\mathcal{M}=(M, u)$ obtained by $C^{2}$-immersion $u: M \rightarrow \mathbb{R}^{n+1}$. Let $\mathcal{N} \subset \mathbb{R}^{n+1}$ be some domain such that $\mathcal{M} \subset \partial \mathcal{N}$ and $\Phi, \Psi: \mathbb{R}^{n+1} \rightarrow \mathbb{R}$ be $C^{2}$-smooth functions. If $\xi$ is a field of unit normals on the surface $\mathcal{M}$, then for each $C^{2}$-smooth surface $\mathcal{M}$ a potential energy functional is well-defined

$$
W(\mathcal{M})=\int_{\mathcal{M}} \Phi(\xi) d \mathcal{M}+\int_{\mathcal{N}} \Psi(x) d x
$$

independent on the choice of the normal $\xi$.

On the surface $\mathcal{M}$, a Riemannian metrics and an associated scalar product of tangential vectors are induced; we shall denote the latter scalar product as the scalar product in $\mathbb{R}^{n+1}$ by $\langle\cdot, \cdot\rangle$. The symbols $\bar{\nabla}$ and $\nabla$ stand for the Riemmanian connectivities in $\mathbb{R}^{n+1}$ and $\mathcal{M}$, repspectively. The following relations are known [10]

$$
\nabla h=(\bar{\nabla} h)^{T}, \quad \nabla_{X} Y=\left(\bar{\nabla}_{X} Y\right)^{T}
$$


to hold for arbitrary $C^{1}$-smooth functions $h: \mathbb{R}^{n+1} \rightarrow \mathbb{R}$ and for $C^{1}$-smooth vector fields $X$ and $Y$ tangential to $\mathcal{M}$. By the symbol $v^{T}$ we denote an orthogonal projection of the vector $v$ on the tangential plane $T_{m} \mathcal{M}$ to the surface $\mathcal{M}$ at a corresponding point $m \in \mathcal{M}$. Then the divergence of the vector field $X$, as a section of tangential bundle of the surface $\mathcal{M}$ is determined as a trace of linear mapping $E \rightarrow \nabla_{E} X$ [9. Ch. VI, Sect. 4]. Choosing an orthonormalized basis $\left\{Z_{i}\right\}_{i=1}^{n}$ in the tangential space $T_{m} \mathcal{M}$, according [9, Ch. VI, Sect. 5], the divergence of a vector field $X$ can be written as

$$
\operatorname{div} X=\sum_{i=1}^{n}\left\langle\nabla_{Z_{i}} X, Z_{i}\right\rangle .
$$

Let $m \in \mathcal{M}$ and let in some neighbourhood of a point $u(m)$ smooth vector fields $X$ and $Y$ be defined. Then a bilinear form

$$
B(X(m), Y(m))=\left(\bar{\nabla}_{X} Y\right)(u(m))-\left(\bar{\nabla}_{X} Y\right)^{T}(u(m))
$$

is called a second fundamental form of the surface $\mathcal{M}$, see [10]. The form $B(X, Y)$ is symmetric [10, Ch. VI, Sect. 3]. For a chosen orthonormalized basis in the tangential space $T_{m} \mathcal{M}$ to the surface $\mathcal{M}$ at a point $u(m)$, the vector

$$
\vec{H}(m)=\frac{1}{n} \operatorname{tr} B=\frac{1}{n} \sum_{i=1}^{n} B\left(Z_{i}, Z_{i}\right)
$$

is called mean curvature normal [10, Ch. VII, Sect. 5] of surface $\mathcal{M}$ at the point $u(m)$.

Let $V$ be $C^{2}$-vector field defined in a neighbourhood of the surface $\mathcal{M}$ such that $\left.V\right|_{\mathcal{M}}=h \cdot \xi$, where $h \in C_{0}^{1}(\mathcal{M})$, and $\xi$ be the field of unit normals to the surface and at that it is assumed that the integral curves of the field $V$ are located on straight lines and along these lines the identity $|V|=$ const holds.

It is clear that if the surface $\mathcal{M}$ is immersed, then each vector field $V=h \cdot \xi$ defined along $\mathcal{M}$ can be continued into some neighbourhood $\mathcal{M}$ so that the aforementioned conditions are satisfied. We note that according work [2], the second variation is independent of the choice of continuation.

Let $U(\mathcal{M})$ be a neighbourhood of the surface $\mathcal{M}$, in which the field $V$ is defined and $g_{t}(x)$ : $U(\mathcal{M}) \rightarrow \mathbb{R}^{n+1}$ be a one-parametric group of local diffeomorphisms generated by the vector field $V$. That is, $g_{t}(x)$ is a solution to the Cauchy problem:

$$
\frac{d g_{t}(x)}{d t}=V\left(g_{t}(x)\right),\left.\quad g_{t}(x)\right|_{t=0}=x .
$$

We let $\mathcal{M}_{t}=g_{t}(\mathcal{M})$ and $\mathcal{N}_{t}=g_{t}(\mathcal{N})$. It is clear that $\mathcal{M}_{0}=\mathcal{M}, \mathcal{N}_{0}=\mathcal{N}$.

Definition 2.1. A surface $\mathcal{M}$ is extremal if the second variation of functional (2.1) vanishes for all infinitesimal deformations of the surface $\mathcal{M}$, that is,

$$
\left.\frac{d W(t)}{d t}\right|_{t=0}=\left.\frac{d}{d t}\left(\int_{\mathcal{M}_{t}} \Phi(\xi) d \mathcal{M}_{t}+\int_{\mathcal{N}_{t}} \Psi(x) d x\right)\right|_{t=0}=0 .
$$

Definition 2.2. An extremal surface $\mathcal{M}$ is stable if the second variation of functional (2.1) is sign-definite for all infinitesimal deformations of the surface $\mathcal{M}$, otherwise it is unstable.

Definition 2.3. A surface $\mathcal{M}$ is tubular [7] if there exist two numbers $-\infty \leqslant a<b \leqslant+\infty$ such that for each hyperplane

$$
\Pi_{t}=\left\{x \in \mathbb{R}^{n+1}: x_{n+1}=t\right\}
$$


orthogonal to the vector $e_{n+1} \in \mathbb{R}^{n+1}$, the section $\Sigma(t)=\mathcal{M} \cap \Pi_{t}$ is non-empty for all $t \in(a ; b)$ and each portion between two hyperplanes $\Pi_{t_{1}}$ and $\Pi_{t_{2}}$ for $a<t_{1}<t_{2}<b$ is a compact set.

The surface $\mathcal{M}$ is totally tubular if $a=-\infty$ and $b=+\infty$. The simplest example of totally tubular surface in $\mathbb{R}^{3}$ is catenoid.

The elements of the matrices $G$ are of form

$$
G_{i j}=\frac{\partial^{2} \Phi}{\partial \xi_{i} \partial \xi_{j}}+\delta_{i j}(\Phi-\langle D \Phi, \xi\rangle)
$$

where $D \Phi=\left(\frac{\partial \Phi}{\partial \xi_{1}}, \frac{\partial \Phi}{\partial \xi_{2}}, \ldots, \frac{\partial \Phi}{\partial \xi_{n+1}}\right)$, where $\delta_{i j}$ is the Kronecker delta.

We shall employ the following notations: $k_{i}$ are the principal curvature, $E_{i}$ are principal directions of a surface, div is the divergence in the metrics of a surface $\mathcal{M}$, and $H=\langle\vec{H}, \xi\rangle$ is the mean curvature of a surface $\mathcal{M}$ with respect to the normal $\xi$.

Then the following theorem on variations of the potential energy functional hold.

Theorem 2.1. If $W(t)=W\left(\mathcal{M}_{t}\right)$, then

$$
W^{\prime}(0)=\int_{\mathcal{M}}\left(\operatorname{div}(D \Phi(\xi))^{T}-n H \Phi(\xi)+\Psi(x)\right) h(x) d \mathcal{M} .
$$

Moreover, if $W^{\prime}(0)=0$ for each function $h(x) \in C_{0}^{1}(\mathcal{M})$, then the identity holds:

$$
W^{\prime \prime}(0)=\int_{\mathcal{M}}\left\{G(\nabla h, \nabla h)+h^{2}\left(\langle\bar{\nabla} \Psi(x), \xi\rangle-\sum_{i=1}^{n} k_{i}^{2} G\left(E_{i}, E_{i}\right)\right)\right\} d \mathcal{M},
$$

where $G$ is a quadratic form associated with matrix $(2.2)$.

Formula (2.3) for the first variation of functional (2.1) was proved in [11], while formula (2.4) for the second variation was established in work [12].

\section{THEOREM ON INSTABILITY OF TUBUlAR SURFACES}

Hereafter we assume that the matrix $G$ in $(2.2)$ is positive definite, $\lambda(\xi)$ and $\Lambda(\xi)$ are the eigenvalues of the matrix $G$ with the minimal and maximal absolute values.

In order to prove the theorem on instability, we shall employ an extremal property of eigenvalues of positive definite matrices [13. In the general case we have

$$
\lambda(\xi)|\eta|^{2} \leqslant G(\eta, \eta) \leqslant \Lambda(\xi)|\eta|^{2}
$$

for all vectors $\xi, \eta \in \mathbb{R}^{n+1}$, where the matrix $G$ is evaluated at the point $\xi$. In particular, this implies

$$
\lambda(\xi)|\nabla h|^{2} \leqslant G(\nabla h, \nabla h) \leqslant \Lambda(\xi)|\nabla h|^{2}
$$

and

since $\left|E_{i}\right|^{2}=1$.

$$
\lambda(\xi) \leqslant G\left(E_{i}, E_{i}\right) \leqslant \Lambda(\xi)
$$

Assume that along the surface the condition

$$
\langle\bar{\nabla} \Psi, \xi\rangle \leqslant 0
$$

holds. In fact, it relates the loads on the surface from outside and inside. The author does not know the interpretation of this condition in application and it is needed exclusively because of features of the method of proving, similar to work [4]. 
Let $L_{\min }, L_{\max }$ be the minimal and maximal $(n-1)$-dimensional measures of the section of surface $\mathcal{M}$ by the planes of form $\left\{x: x_{n+1}=\right.$ const $\}$ and

$$
\Lambda^{2}=\max _{\mathcal{M}} \Lambda(\xi), \quad \lambda^{2}=\min _{\mathcal{M}} \lambda(\xi), \quad H_{\min }=\min _{\mathcal{M}}|H| .
$$

The following theorem holds.

Theorem 3.1. Let $\mathcal{M}$ be an immersed tubular surface extremal for functional (1), with mean curvature $H_{\min } \neq 0$ in $\mathbb{R}^{n+1}$, along which the inequality $\langle\bar{\nabla} \Psi, \xi\rangle \leqslant 0$ holds and the edge of the surface is located in the parallel planes $\alpha_{1}$ and $\alpha_{2}$ defined by the equations $x_{n+1}=a$ and $x_{n+1}=b$, respectively. If the distance between these planes satisfies the inequality

$$
b-a>\frac{2 \pi \Lambda L_{\max }}{\sqrt{n} H_{\min } \lambda L_{\min }},
$$

then the surface $\mathcal{M}$ is unstable.

Remark 3.1. A similar theorem for the surfaces of constant mean curvature was proved by V.A. Klyachin in 4].

Proof. In order to prove the instability of a surface, it is sufficient to find a function $h: \mathcal{M} \rightarrow \mathbb{R}$, for which the second variation of the functional is negative as it is required by the definition of the instability; we recall that we consider only positive definite matrices $G$. Since expression (2.4) for the second variation holds for each function $h \in C_{0}^{1}(\mathcal{M})$, for the sake of brevity it is necessary to clarify that a condition for the function $h(x)$ is chosen by the identities

$$
\left.h(x)\right|_{\partial \mathcal{M}}=0, \quad \int_{\mathcal{M}} h(x) d \mathcal{M}=0
$$

for the cases of non-empty and empty boundary of the surface $\mathcal{M}$, respectively. Let us estimate the second variation taking into consideration the conditions (3.1), (3.2), (3.3) and $\|A\|^{2}=$ $\sum_{i=1}^{n} k_{i}^{2}$. We have:

$$
\begin{aligned}
I(h) & =\int_{\mathcal{M}}\left(G(\nabla h, \nabla h)+h^{2}\left(\langle\bar{\nabla} \Psi, \xi\rangle-\sum_{i=1}^{n} k_{i}^{2} G\left(E_{i}, E_{i}\right)\right)\right) d \mathcal{M} \\
& \leqslant \int_{\mathcal{M}}\left(G(\nabla h, \nabla h)-h^{2} \sum_{i=1}^{n} k_{i}^{2} G\left(E_{i}, E_{i}\right)\right) d \mathcal{M} \leqslant \int_{\mathcal{M}}\left(\Lambda(\xi)|\nabla h|^{2}-h^{2} \lambda(\xi)\|A\|^{2}\right) d \mathcal{M} .
\end{aligned}
$$

Let

$$
\Sigma(t)=\{x \in \mathcal{M}: f(x)=t\}=\mathcal{M} \bigcap\left\{x: x_{n+1}=t\right\}, \quad f(x)=x_{n+1} .
$$

We seek a needed function $h$ as $h=h(f(x))$.

According Kronrod-Federer formula [14, Thm. 3.2.12], inequality $\|A\|^{2} \geqslant n H_{\text {min }}^{2}$ and the introduced notations $\Lambda^{2}=\max _{\mathcal{M}} \Lambda(\xi), \lambda^{2}=\min _{\mathcal{M}} \lambda(\xi)$, we have

$$
\begin{aligned}
I(h) & \leqslant \int_{\mathcal{M}}\left(\Lambda(\xi)|\nabla h|^{2}-h^{2} \lambda(\xi)\|A\|^{2}\right) d \mathcal{M} \\
& \leqslant \Lambda^{2} \int_{a}^{b}\left(h^{\prime}\right)^{2}(\tau) \int_{\Sigma(\tau)}|\nabla f| d \Sigma(\tau) d \tau-n H_{\min }^{2} \lambda^{2} \int_{a}^{b} h^{2}(\tau) \int_{\Sigma(\tau)} \frac{1}{|\nabla f|} d \Sigma(\tau) d \tau .
\end{aligned}
$$


We introduce shorthand notations

$$
\eta(\tau)=\int_{\Sigma(\tau)}|\nabla f| d \Sigma(\tau), \quad \nu(\tau)=\int_{\Sigma(\tau)} \frac{1}{|\nabla f|} d \Sigma(\tau)
$$

and $d \Sigma(t)$ is the area element of the section $\Sigma(t)$. Then inequality becomes

$$
I(h) \leqslant \Lambda^{2} \int_{a}^{b}\left(h^{\prime}\right)^{2}(\tau) \eta(\tau) d \tau-n H_{\min }^{2} \lambda^{2} \int_{a}^{b} h^{2}(\tau) \nu(\tau) d \tau .
$$

We let

$$
h(t)= \begin{cases}\sin \left(\frac{2 \pi \sigma}{\sigma_{0}}\right), & 0 \leqslant \sigma<\frac{\sigma_{0}}{2} \\ \mu \sin \left(\frac{2 \pi \sigma}{\sigma_{0}}\right), & \frac{\sigma_{0}}{2} \leqslant \sigma \leqslant \sigma_{0}\end{cases}
$$

where

$$
\sigma(t)=\int_{a}^{t} \frac{d \tau}{\eta(\tau)}, \quad \sigma_{0}=\int_{a}^{b} \frac{d \tau}{\eta(\tau)}
$$

$\mu$ is a positive constant, which is chosen in view of 3.4 :

$$
\int_{\mathcal{M}} h(x) d \mathcal{M}=\int_{a}^{b} h(\tau) \nu(\tau) d \tau=0 .
$$

The existence of such constant is obvious by construction of the function $h(t)$.

We find the derivatives:

$$
h^{\prime}(t)= \begin{cases}\frac{2 \pi}{\sigma_{0}} \cos \left(\frac{2 \pi \sigma}{\sigma_{0}}\right) \sigma^{\prime}(t)=\frac{2 \pi}{\sigma_{0}} \cos \left(\frac{2 \pi \sigma}{\sigma_{0}}\right) \frac{1}{\eta(t)}, & 0 \leqslant \sigma<\frac{\sigma_{0}}{2}, \\ \mu \frac{2 \pi}{\sigma_{0}} \cos \left(\frac{2 \pi \sigma}{\sigma_{0}}\right) \sigma^{\prime}(t)=\mu \frac{2 \pi}{\sigma_{0}} \cos \left(\frac{2 \pi \sigma}{\sigma_{0}}\right) \frac{1}{\eta(t)}, & \frac{\sigma_{0}}{2} \leqslant \sigma \leqslant \sigma_{0} .\end{cases}
$$

Under the change of variable $d \sigma(t)=d t / \eta(t)$, the inequality becomes:

$$
\begin{aligned}
I(h) \leqslant & \left(\frac{2 \pi}{\sigma_{0}}\right)^{2} \Lambda^{2} \int_{0}^{\frac{\sigma_{0}}{2}} \cos ^{2}\left(\frac{2 \pi \sigma}{\sigma_{0}}\right) d \sigma-n H_{\min }^{2} \lambda^{2} \int_{0}^{\frac{\sigma_{0}}{2}} \sin ^{2}\left(\frac{2 \pi \sigma}{\sigma_{0}}\right) \nu(\tau(\sigma)) \eta(\tau(\sigma)) d \sigma \\
& +\mu^{2}\left(\frac{2 \pi}{\sigma_{0}}\right)^{2} \Lambda^{2} \int_{\frac{\sigma_{0}}{2}}^{\sigma_{0}} \cos ^{2}\left(\frac{2 \pi \sigma}{\sigma_{0}}\right) d \sigma-\mu^{2} n H_{\min }^{2} \lambda^{2} \int_{\frac{\sigma_{0}}{2}}^{\sigma_{0}} \sin ^{2}\left(\frac{2 \pi \sigma}{\sigma_{0}}\right) \nu(\tau(\sigma)) \eta(\tau(\sigma)) d \sigma .
\end{aligned}
$$

By the Cauchy-Schwarz inequality, the $(n-1)$-dimensional area $L(t)$ of section $\Sigma(t)$ can be estimated as

$$
L(t)=\int_{\Sigma(t)} d \Sigma(t) \leqslant\left(\int_{\Sigma(\tau)}|\nabla f| d \Sigma(\tau)\right)^{\frac{1}{2}}\left(\int_{\Sigma(\tau)} \frac{1}{|\nabla f|} d \Sigma(\tau)\right)^{\frac{1}{2}}=\sqrt{\eta(t) \nu(t)} .
$$


This is why, letting $L_{\max }=\max _{[a, b]} L(t), L_{\min }=\min _{[a, b]} L(t)$, we get:

$$
\begin{aligned}
I(h) \leqslant & \left(\frac{2 \pi}{\sigma_{0}}\right)^{2} \Lambda^{2} \int_{0}^{\frac{\sigma_{0}}{2}} \cos ^{2}\left(\frac{2 \pi \sigma}{\sigma_{0}}\right) d \sigma-L_{\min }^{2} n H_{\min }^{2} \lambda^{2} \int_{0}^{\frac{\sigma_{0}}{2}} \sin ^{2}\left(\frac{2 \pi \sigma}{\sigma_{0}}\right) d \sigma \\
& +\mu^{2}\left(\frac{2 \pi}{\sigma_{0}}\right)^{2} \Lambda^{2} \int_{\frac{\sigma_{0}}{2}}^{\sigma_{0}} \cos ^{2}\left(\frac{2 \pi \sigma}{\sigma_{0}}\right) d \sigma-L_{\min }^{2} \mu^{2} n H_{\min }^{2} \lambda^{2} \int_{\frac{\sigma_{0}}{2}}^{\sigma_{0}} \sin ^{2}\left(\frac{2 \pi \sigma}{\sigma_{0}}\right) d \sigma .
\end{aligned}
$$

Calculating the integrals, we obtain that they are equal to

$$
\begin{array}{ll}
\int_{0}^{\frac{\sigma_{0}}{2}} \cos ^{2}\left(\frac{2 \pi \sigma}{\sigma_{0}}\right) d \sigma=\frac{\sigma_{0}}{4}, & \int_{\frac{\sigma_{0}}{2}}^{\sigma_{0}} \cos ^{2}\left(\frac{2 \pi \sigma}{\sigma_{0}}\right) d \sigma=\frac{\sigma_{0}}{4}, \\
\int_{0}^{\frac{\sigma_{0}}{2}} \sin ^{2}\left(\frac{2 \pi \sigma}{\sigma_{0}}\right) d \sigma=\frac{\sigma_{0}}{4}, & \int_{\frac{\sigma_{0}}{2}}^{\sigma_{0}} \sin ^{2}\left(\frac{2 \pi \sigma}{\sigma_{0}}\right) d \sigma=\frac{\sigma_{0}}{4},
\end{array}
$$

and this is the inequality casts into the form:

$$
I(h) \leqslant \frac{\sigma_{0}}{4}\left(1+\mu^{2}\right)\left(\left(\frac{2 \pi}{\sigma_{0}}\right)^{2} \Lambda^{2}-n H_{\min }^{2} L_{\min }^{2} \lambda^{2}\right) .
$$

Recalling that

$$
\sigma_{0}=\int_{a}^{b} \frac{d t}{\eta(t)} \geqslant \int_{a}^{b} \frac{d t}{L(t)} \geqslant \frac{b-a}{L_{\max }}
$$

we finally get:

$$
I(h) \leqslant \frac{\sigma_{0}}{4}\left(1+\mu^{2}\right) L_{\max }^{2}\left(\left(\frac{2 \pi}{b-a}\right)^{2} \Lambda^{2}-n H_{\min }^{2} \frac{L_{\min }^{2}}{L_{\max }^{2}} \lambda^{2}\right) .
$$

By the assumptions of the theorem, the surface is unstable and this implies that the second variation is negative. Thus,

$$
\left(\frac{2 \pi}{b-a}\right)^{2} \Lambda^{2}-n H_{\min }^{2} \frac{L_{\min }^{2}}{L_{\max }^{2}} \lambda^{2}<0 .
$$

Then, expressing the difference $b-a$ from this inequality, we obtain the estimate from the formulation of the theorem:

$$
b-a>\frac{2 \pi \Lambda L_{\max }}{\sqrt{n} H_{\min } \lambda L_{\min }} .
$$

The proof is complete. 


\section{BIBLIOGRAPHY}

1. R. Finn. Equilibrium capillary surfaces. Springer-Verlag, New York (1986).

2. J. Simons. Minimal varieties in riemannian manifolds // Ann. Math. 88:1, 62-105 (1968).

3. V.A. Klyachin, V.M. Miklyukov. Criteria of instability of surfaces of zero mean curvature in warped Lorentz products // Matem. Sborn. 187:11, 67-88 (1996). [Sb. Math. 187:11, 1643-1663 (1996).]

4. V.A. Klyachin. On some properties of stable and unstable surfaces with prescribed mean curvature // Izv. RAN. Ser. Matem. 70:4, 77-90 (2006). [Izv. Math. 70:4, 77-90 (2006)].

5. V.A. Saranin. Equilibrium of liquids and its stability. Inst. Comp. Studies, Moscow (2002). (in Russian).

6. N.M. Medvedeva. Research of stability for extremal rotation surfaces // Izv. Saratov Univ. (N.S.). Ser. Math. Mech. Inform. 7:2, 25-32 (2007).

7. V.M. Miklyukov, V.G. Tkachev. Some properties of the tubular minimal surfaces of arbitrary codimension // Matem. Sborn. 180:9, 1278-1295 (1989). [Math. USSR-Sb. 68:1, 133-150 (1991).]

8. N.M. Poluboyarova. Instability of tubular extremal surfaces // in Proc. Int. Conf. "Complex analysis and geometry", Ufa, 32 (2018). (in Russian).

9. Sh. Kobayashi, K. Nomizu. Foundations of differential geometry. V. 1. Interscience Publ., a division of John Wiley \& Sons, New York (1963).

10. Sh. Kobayashi, K. Nomizu. Foundations of differential geometry. V. 2. Interscience Publ., a division of John Wiley \& Sons, New York (1969).

11. N.M. Poluboyarova. Equations of extremals of potential energy // Vestn. Volgograd. Gosud. Univ. Ser. 1. Matem. Fiz. 5(36), 60-72 (2016). (in Russian).

12. N.M. Poluboyarova. On instability of extremals of potential energy functional // Ufimskij Matem. Zhurn. 10:3, 79-88 (2018). [Ufa Math. J. 10:3, 77-85 (2018).]

13. I.M. Gel'fand. Lectures on linear algebra. Nauka, Moscow (1971). [Robert E. Krieger Publ. Co., New York (1978).]

14. H. Federer. Geometric measure theory. Springer-Verlag, Berlin (1987).

too long tubes with nonzero mean curvature are instable.

Natalia Mikhailovna Poluboyarova,

Volgograd State University,

Universitetsky av. 100,

400062, Volgograd, Russia

E-mail: natasha_medvedeva@volsu.ru 